\section{Iniquidades no acesso aos benefícios alimentação e refeição no Brasil: uma análise da Pesquisa de Orçamentos Familiares 2008-2009}

\author{
Inequities in access to food stamps and meal \\ vouchers in Brazil: an analysis of the Brazilian \\ Household Budgets Survey, 2008-2009
}

\section{La inequidad en el acceso a los vales de alimentos y comida en Brasil: un análisis de las Encuestas de Presupuestos Familiares 2008-2009}

\section{Resumo}

Considerando que o recebimento de benefícios alimentação e refeição pode ser um determinante da escolha alimentar de trabalhadores, o objetivo do estudo foi avaliar a abrangência do recebimento destes benefícios no Brasil e sua distribuição segundo características sociodemográficas e regionais dos beneficiários. Utilizaram-se dados da Pesquisa de Orçamentos Familiares 2008-2009. Foram elegíveis os indivíduos que referiram ter ocupação e declararam possuir emprego privado, público, doméstico ou temporário na área rural. Apenas $3,2 \%$ dos elegíveis referiram receber os benefícios, com maior concentração no Sudeste, área urbana, sexo masculino, empregados no setor privado e recebendo mais de 5 salários mínimos. O valor médio mensal dos benefícios recebidos no Brasil foi $R \$ 177,20$. Após ajuste para as demais variáveis, o valor foi maior entre homens, com salários mais altos, no Nordeste e Centro-oeste e do setor público. Trata-se da primeira análise de abrangência nacional sobre benefícios alimentação e refeição, apontando uma importante parcela de trabalhadores sem acesso ao benefício e iniquidades no acesso.

Assistência Alimentar; Trabalhadores; Alimentação Coletiva; Programas e Políticas de Nutrição e Alimentação; Iniquidade Social
Daniela Silva Canella 1,2 Ana Paula Bortoletto Martins 2 Daniel Henrique Bandoni 2,3

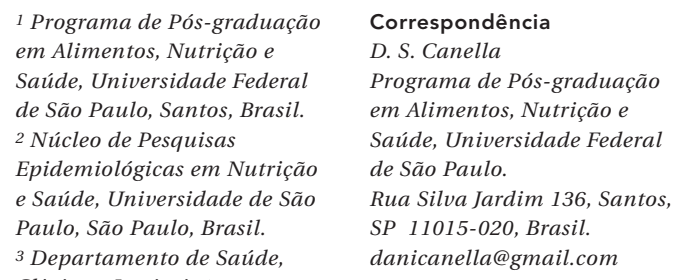

\author{
Correspondência \\ D. S. Canella \\ Programa de Pós-graduação \\ em Alimentos, Nutrição e \\ Saúde, Universidade Federal \\ de São Paulo. \\ Rua Silva Jardim 136, Santos, \\ SP 11015-020, Brasil. \\ danicanella@gmail.com
}




\section{Introdução}

O padrão de alimentação no Brasil sofreu intensa e rápida modificação nas últimas décadas. A tendência observada por meio de inquéritos nacionais evidencia aumento expressivo no consumo de carnes, leite e derivados, e óleos vegetais, substancial redução no consumo de cereais, leguminosas e tubérculos, além do constante baixo consumo de frutas e hortaliças 1,2,3,4.

Algumas das causas responsáveis pelas recentes mudanças na alimentação são complexas, ainda pouco estudadas e envolvem diferentes aspectos como: mudanças tecnológicas, políticas agrícolas, novas formas de distribuição e marketing dos alimentos, variações na renda das famílias, tendências dos preços relativos dos diferentes alimentos e conhecimento dos indivíduos sobre alimentação e saúde 5. Outra expressiva mudança nos padrões de alimentação dos brasileiros foi em relação ao local de realização das refeições. Entre 2002-2003 e 2008-2009, a participação da alimentação fora do domicílio cresceu $25 \%$, chegando a representar $28 \%$ dos gastos totais com alimentação e $18 \%$ da energia consumida diariamente 6,7 .

Nesse cenário, o recebimento de benefícios alimentação e refeição é um importante determinante da escolha alimentar, podendo ser utilizado para aquisição de alimentos em supermercados ou de refeições em restaurantes comerciais 8 . Esse tipo de benefício geralmente é oferecido por empresas a seus trabalhadores formalizados, que realizam suas refeições fora do local de trabalho, pagando por meio de um cartão operado por redes independentes, que assumem a tarefa de interligar estabelecimentos comerciais e trabalhadores.

Um grande impulso no acesso aos benefícios alimentação e refeição ocorreu a partir da década de 1980, quando esta modalidade de benefício passou a integrar o Programa de Alimentação do Trabalhador (PAT), somando-se às modalidades de oferta de refeições no local de trabalho e de cesta de alimentos, política federal que concede incentivo fiscal às empresas que fornecem alimentação aos seus trabalhadores ${ }^{9}$. Assim, esses benefícios podem ser considerados alguns dos responsáveis pela grande expansão do PAT, favorecendo a entrada de empresas de menor porte no programa, as quais não teriam estrutura para produzir refeições no ambiente de trabalho. Entretanto, essa modalidade de benefício tem acarretado problemas passíveis de críticas, como a falta de pessoal para fiscalizar os restaurantes credenciados, o que implica ausência de controle sobre a qualidade das refeições servidas, desvios e fraudes 10 . Destaca-se que, ainda que essa seja uma das modalidades do PAT, empresas podem oferecer os benefícios alimentação e refeição a seus trabalhadores sem estarem cadastradas no programa.

Dados do Ministério do Trabalho e Emprego mostram que, em 2014, mais de 18 milhões de trabalhadores participavam do PAT, destes, 37\% recebiam benefício alimentação e $23 \%$ benefício refeição, no entanto, é possível que existam sobreposições nestes dados com um mesmo trabalhador recebendo mais de um benefício. Assim, esses auxílios representam cerca de $60 \%$ dos benefícios fornecidos pelas empresas cadastradas no programa 9 . Ademais, de acordo com a Associação Brasileira das Empresas de Refeições Coletivas (ABERC), o benefício refeição foi utilizado em 2014 para pagar diariamente 7,4 milhões de refeições consumidas fora de casa, representando mais de um terço do total destas refeições no país 11 .

Ainda que não se tenha dados ou estimativas acurados disponíveis que quantifiquem o total de indivíduos beneficiados, cerca de $58 \%$ da população brasileira maior de 16 anos estão ocupadas em trabalho formal e poderiam receber os benefícios alimentação ou refeição 12 .

Apesar da importância de benefícios do tipo alimentação e refeição, poucos estudos têm caracterizado sua população beneficiária. Diante desse quadro, o presente estudo pretendeu avaliar a abrangência dos benefícios alimentação e refeição no Brasil, sua distribuição segundo as características sociodemográficas e regionais, e avaliar o estado nutricional dos beneficiários.

\section{Métodos}

Para o presente trabalho foram utilizados dados da Pesquisa de Orçamentos Familiares (POF), realizada pelo Instituto Brasileiro de Geografia e Estatística (IBGE) entre 19 de maio de 2008 e 18 de maio de 2009. Trata-se de uma pesquisa com dados representativos da população brasileira, que envolveu entrevistas realizadas em uma amostra de 55.970 domicílios, distribuídos entre as cinco macrorregiões e as áreas urbana e rural 13.

A POF 2008-2009 utilizou plano amostral complexo, por conglomerados, envolvendo estratificação geográfica e socioeconômica de todos os setores censitários do país, seguida de sorteios aleatórios de setores no primeiro estágio e de domicílios no segundo. A coleta de dados foi realizada ao longo de 12 meses, de maneira uniforme nos estratos, garantindo a representatividade nos quatro trimestres do ano. As características do processo de amostragem da POF estão descritas em detalhe em publicação do IBGE 13. 
A principal informação da POF 2008-2009 utilizada no presente estudo refere-se ao recebimento de benefícios alimentação e refeição, nos últimos 12 meses (em valor mensal). Adicionalmente, utilizaram-se dados referentes aos indivíduos (sexo, idade, tipo de ocupação, salário e estado nutricional) e aos domicílios (região e área).

Para este trabalho, foram incluídos apenas os indivíduos que referiram possuir uma ocupação, sendo elegíveis aqueles que se declararam: empregado privado, empregado público, empregado doméstico ou empregado temporário na área rural.

Entre esses, selecionaram-se os que informaram o recebimento de benefícios alimentação e refeição. Na coleta de dados da POF, os dois benefícios foram registrados em conjunto e chamados de "auxílio alimentação". Tais informações foram registradas em questionário específico sobre trabalho e rendimento individual, por cada um dos membros do domicílio. Com base nos valores registrados, calculou-se o valor deflacionado dos rendimentos, em Reais (R\$), utilizando-se o Índice Nacional de Preços ao Consumidor Amplo (IPCA), tendo como data de referência o dia 15 de janeiro de 200913.

Foi calculado o valor médio dos benefícios alimentação e refeição recebido (em R\$) e a distribuição das pessoas beneficiadas segundo as características sociodemográficas e os respectivos intervalos de $95 \%$ de confiança. As características sociodemográficas estudadas foram: sexo, idade (até 29 anos, de 30-49 e 50 ou mais), tipo de ocupação (privada ou pública), faixa salarial (categorizada em até 5 salários mínimos, entre 5 e 10 ou acima de 10 salários mínimos); e as características regionais foram: região do Brasil (Norte, Nordeste, Sul, Sudeste e Centro-oeste) e área (urbana ou rural). Ainda, investigou-se o estado nutricional dos beneficiários, classificados de acordo com o seu índice de massa corporal (IMC) em eutróficos ou com baixo peso (IMC $\leq$ $24,99 \mathrm{~kg} / \mathrm{m}^{2}$ ) ou com excesso de peso (IMC $\geq$ $\left.25 \mathrm{~kg} / \mathrm{m}^{2}\right) 14$.

Analisou-se também a associação entre o valor dos benefícios alimentação e refeição (em R\$) e as características sociodemográficas e regionais estudadas por meio de modelos de regressão linear simples e múltiplo. Foram incluídas no modelo múltiplo todas as variáveis que apresentam $\mathrm{p}<0,05$ nos modelos simples.

Todas as análises foram realizadas com o emprego do pacote estatístico Stata/SE versão 12.1 (StataCorp LP, College Station, Estados Unidos), no módulo survey, que considera os efeitos da amostragem complexa e permite a extrapolação dos resultados para a população brasileira, considerando o nível de 5\% de significância .
Este estudo foi aprovado pelo Comitê de Ética em Pesquisa da Universidade Federal de São Paulo (Parecer no 965.555).

\section{Resultados}

Ao todo a POF 2008-2009 estudou 190.159 indivíduos de todas as idades, dos quais 101.053 declararam alguma ocupação e 62.275 eram elegíveis para o estudo. Desses, 2.008 indivíduos $(3,2 \%$ da população ocupada) referiram receber benefícios alimentação e refeição. Na Tabela 1, observa-se que há uma concentração de beneficiários na Região Sudeste (63,3\%), na área urbana $(95,2 \%)$, entre indivíduos do sexo masculino (63\%), empregados do setor privado $(73,5 \%)$ e recebendo mais de cinco salários mínimos (74,3\%).

$\mathrm{O}$ valor mensal médio do benefício recebido no Brasil foi R\$ 177,20 (IC95\%: R\$ 166,08; R\$ $188,31)$. Destaca-se que o maior valor médio recebido foi entre os trabalhadores residentes na Região Centro-oeste (R\$232,46), na área urbana $(\mathrm{R} \$ 178,75)$, empregados no setor público (R\$ $227,28)$ e que recebiam 10 ou mais salários mínimos (R \$ 239,39). Os trabalhadores das regiões Sul e Sudeste receberam um valor médio abaixo da média nacional (Tabela 1).

Em relação ao estado nutricional dos beneficiários, verificou-se que 53\% (IC95\%: 49,75\%; $56,27 \%$ ) dos indivíduos apresentaram excesso de peso, sendo os demais eutróficos ou com baixo peso (47\% - IC95\%: 43,73\%; 50,25\%). Ambos os grupos referiram receber valores médios semelhantes: R\$ 176,85 (IC95\%: R\$ 161,51; R\$ 192,18) e R\$ 178,21 (IC95\%: R\$ 165,60; R\$ 190,81), respectivamente. Destaca-se que apenas $2,3 \%$ dos pesquisados apresentaram baixo peso, assim, optou-se por analisá-los em conjunto com os indivíduos eutróficos.

Na Tabela 2, estão descritos os resultados dos modelos de regressão simples e ajustados para a relação entre o valor do benefício e as variáveis sociodemográficas e regionais. Observa-se que, após ajuste para as demais variáveis, o valor do benefício alimentação e refeição foi maior conforme o aumento salarial. O valor do benefício também foi superior nas regiões Nordeste e Centro-oeste, entre empregados do setor público e para os homens.

\section{Discussão}

A análise do recebimento de benefícios alimentação e refeição pela população brasileira ocupada, com base nos dados da POF 2008-2009, 
Distribuição (em \%) dos indivíduos que receberam benefícios alimentação e refeição e dos valores médios mensais (em $\mathrm{R} \$$ ) recebidos, segundo características sociodemográficas e regionais. Brasil, 2008-2009.

\begin{tabular}{|c|c|c|c|c|}
\hline Características & $\%$ & IC95\% & $\begin{array}{c}\text { Valor mensal } \\
\quad(e m \mathrm{R} \$)\end{array}$ & IC95\% \\
\hline \multicolumn{5}{|l|}{ Região } \\
\hline Norte & 4,13 & 3,$52 ; 4,74$ & 212,11 & 179,$93 ; 244,28$ \\
\hline Nordeste & 11,84 & 10,$12 ; 13,56$ & 214,60 & 180,$07 ; 249,14$ \\
\hline Sudeste & 63,27 & 60,$17 ; 66,36$ & 163,41 & 148,$72 ; 178,10$ \\
\hline Sul & 15,52 & 13,$70 ; 17,35$ & 176,93 & 158,$24 ; 195,61$ \\
\hline Centro-oeste & 5,24 & 4,$46 ; 6,02$ & 232,46 & 205,$34 ; 259,58$ \\
\hline \multicolumn{5}{|l|}{ Área } \\
\hline Rural & 4,83 & 3,$47 ; 6,20$ & 146,56 & 125,$26 ; 167,87$ \\
\hline Urbana & 95,17 & 93,$80 ; 96,53$ & 178,75 & 167,$07 ; 190,43$ \\
\hline \multicolumn{5}{|l|}{ Sexo } \\
\hline Masculino & 63,02 & 59,$40 ; 66,63$ & 180,50 & 168,$10 ; 192,89$ \\
\hline Feminino & 36,98 & 33,$37 ; 40,60$ & 171,57 & 154,$40 ; 188,74$ \\
\hline \multicolumn{5}{|l|}{ Faixa etária (anos) } \\
\hline$<29$ & 30,69 & 27,$26 ; 34,12$ & 162,34 & 147,$01 ; 177,67$ \\
\hline $30-49$ & 56,60 & 52,$67 ; 60,53$ & 180,46 & 165,$22 ; 195,69$ \\
\hline 50 ou mais & 12,71 & 10,$29 ; 15,13$ & 198,57 & 172,$16 ; 224,97$ \\
\hline \multicolumn{5}{|l|}{ Faixa salarial (salários mínimos) } \\
\hline$<5$ & 25,72 & 22,$57 ; 28,88$ & 123,70 & 114,$08 ; 133,32$ \\
\hline $5-10$ & 34,14 & 30,$15 ; 38,13$ & 144,39 & 132,$40 ; 156,37$ \\
\hline$\geq 10$ & 40,14 & 35,$86 ; 44,42$ & 239,39 & 217,$94 ; 260,83$ \\
\hline \multicolumn{5}{|l|}{ Tipo de ocupação } \\
\hline Empregado do setor privado & 73,49 & 69,$88 ; 77,09$ & 159,13 & 147,$06 ; 171,19$ \\
\hline Empregado do setor público & 26,51 & 22,$91 ; 30,12$ & 227,28 & 205,$76 ; 248,81$ \\
\hline
\end{tabular}

IC95\%: intervalo de 95\% de confiança.

indica que a cobertura deste benefício é restrita, visto que apenas cerca de $3 \%$ da população ocupada referiram recebê-lo. Ainda, a distribuição desse benefício apresenta importantes iniquidades, considerando que seu recebimento e valor variaram em função da região do país, do gênero do beneficiário, do tipo de ocupação e da faixa salarial.

O sistema de benefícios alimentação e refeição foi introduzido no Brasil por uma demanda social na década de 1980, quando foram estabelecidas normas para o funcionamento do sistema de refeição convênio (benefício para aquisição de refeições em restaurantes) dentro do PAT 15. De forma similar a outros países, o benefício está ligado a uma política pública, sendo oferecido como um valor adicional ao salário. Países como Hungria e França também têm programas de incentivo à oferta de benefícios alimentação e refeição para trabalhadores, com subsídios oferecidos pelo Estado e contribuição dos mesmos 16.
A despeito de sua importância, são escassos os estudos disponíveis sobre a abrangência e o valor dos benefícios alimentação e refeição no Brasil. Dados da Pesquisa sobre Padrões de Vida (PPV), realizada em 1996-1997, indicam que os benefícios fornecidos cobriam cerca de $20 \%$ da população adulta das regiões Nordeste e Sudeste, número maior que o observado na POF 20082009. Porém, a PPV estudou apenas duas regiões do país e considerou "vale refeição" e "cesta básica” em conjunto 17 , fatores que podem explicar a diferença com os dados do presente estudo.

Apesar de, segundo o Ministério do Trabalho e Emprego, o auxílio alimentação beneficiar cerca de $60 \%$ dos trabalhadores registrados no PAT, pouco mais de um terço dos trabalhadores formais do Brasil faz parte do Programa, assim é possível justificar parte da baixa cobertura dos benefícios alimentação e refeição no país. A própria distribuição do PAT no território nacional pode favorecer as diferenças encontradas na 
Resultados dos modelos de regressão linear para a associação entre o valor dos benefícios alimentação e refeição e características sociodemográficas e regionais. Brasil, 2008-2009.

\begin{tabular}{|c|c|c|c|c|}
\hline \multirow[t]{2}{*}{ Variáveis } & \multicolumn{2}{|c|}{ Coeficiente bruto } & \multicolumn{2}{|c|}{ Coeficiente ajustado * } \\
\hline & $\beta$ & IC95\% & $\beta$ & IC95\% \\
\hline \multicolumn{5}{|l|}{ Região } \\
\hline Sudeste & 1,00 & - & 1,00 & - \\
\hline Nordeste & $51,20 * \star$ & 13,$66 ; 88,73$ & 44,82 ** & 14,$21 ; 75,43$ \\
\hline Norte & $48,70 * \star$ & 13,$33 ; 84,07$ & 33,78 & $-0,80 ; 68,35$ \\
\hline Sul & 13,52 & $-10,25 ; 37,29$ & 11,00 & $-8,93 ; 30,93$ \\
\hline Centro-oeste & $69,05 * *$ & 38,$20 ; 99,90$ & $58,44 * \star$ & 29,$39 ; 87,50$ \\
\hline \multicolumn{5}{|l|}{ Sexo } \\
\hline Masculino & 1,00 & - & 1,00 & - \\
\hline Feminino & $-8,92$ ** & $-33,56 ;-0,18$ & $-16,87 \star \star$ & $-33,56 ;-0,18$ \\
\hline \multicolumn{5}{|l|}{ Faixa salarial (salários mínimos) } \\
\hline$<5$ & 1,00 & - & 1,00 & - \\
\hline $5-10$ & 20,68 ** & 5,$40 ; 35,97$ & 24,16 ** & 9,$35 ; 38,96$ \\
\hline$\geq 10$ & $115,69 * *$ & 92,$19 ; 139,19$ & 106,96 ** & 84,$56 ; 129,36$ \\
\hline \multicolumn{5}{|l|}{ Tipo de ocupação } \\
\hline Empregado do setor privado & 1,00 & - & 1,00 & - \\
\hline Empregado do setor público & $68,16 * \star$ & 43,$91 ; 92,40$ & $33,17 \star \star$ & 9,$09 ; 57,24$ \\
\hline \multicolumn{5}{|l|}{ Área } \\
\hline Rural & 1,00 & - & 1,00 & - \\
\hline Urbana & $32,19 * *$ & 7,$73 ; 56,65$ & 17,18 & $-2,94 ; 37,29$ \\
\hline \multicolumn{5}{|l|}{ Idade (anos) } \\
\hline$<29$ & 1,00 & - & 1,00 & - \\
\hline $30-49$ & 18,12 & $-2,02 ; 38,25$ & 7,88 & $-11,15 ; 26,90$ \\
\hline 50 ou mais & 36,23 ** & 6,$97 ; 65,48$ & 21,99 & $-1,39 ; 45,37$ \\
\hline
\end{tabular}

IC95\%: intervalo de 95\% de confiança.

* Modelo de regressão linear ajustado para região, área, sexo, faixa salarial, tipo da ocupação e idade;

** Valor de $\mathrm{p}<0,05$

proporção de beneficiários no Sudeste, uma vez que do total de trabalhadores beneficiados pelo Programa, cerca de $63 \%$ encontram-se nesta região, sinalizando, assim, a iniquidade no recebimento do benefício 9,12. Ainda em relação ao Sudeste, apesar desta região concentrar a maior proporção de beneficiários, esta apresentou o menor valor médio do benefício, devido à menor proporção de funcionários públicos na região.

Adicionalmente, dados disponíveis na Pesquisa de Condições de Vida, realizada pela Fundação Sistema Estadual de Análise de Dados (Fundação SEADE), e na PPV, do IBGE, mostram que os benefícios do tipo alimentação (ou supermercado) e refeição têm um perfil distributivo concentrado nos estratos de renda superiores, o que é corroborado pelos nossos resultados, que apontam que $75 \%$ dos beneficiários recebem mais de cinco salários mínimos mensais. Assim, se o objetivo dos formuladores de políticas públicas é beneficiar os assalariados com menores rendimentos, como no caso do PAT, é importante incentivar este tipo de benefício em camadas de menor renda 17,18 .

Os dados da POF não permitem avaliar o valor diário dos benefícios alimentação e refeição, entretanto informações da Pesquisa Refeição ASSERT Preço Médio, 2012 19, que avaliou o valor médio da refeição em diferentes modalidades (comercial ou prato feito; autosserviço por quilo ou preço fixo; executivo; e à la carte) no horário de almoço em estabelecimentos que recebiam vales/tíquetes/cartões refeição, apontam que o preço médio da refeição no país é de $\mathrm{R} \$ 22,37$, variando de R\$19,30, no Nordeste, a R\$23,97, no Sudeste. Destaca-se que o valor médio recebido pelos beneficiários pode não ser suficiente para as despesas com alimentação fora do lar 
diariamente, mesmo considerando a refeição comercial ou o prato feito, que apresenta o menor valor médio no país (R\$ 13,07) 19 .

As diferenças observadas na proporção de beneficiários e o valor dos benefícios alimentação e refeição entre os sexos refletem a iniquidade de gênero no mercado de trabalho nacional. Os homens representam mais da metade da população ocupada no país e com maior proporção de trabalhos formais que as mulheres. Adicionalmente, o rendimento médio das mulheres ocupadas corresponde a $75 \%$ do rendimento dos homens nos trabalhos formais e $65 \%$ nos trabalhos informais 12 .

Ainda que o recebimento de benefícios alimentação e refeição não seja restrito a beneficiários do PAT, não devendo necessariamente seguir suas normas, foi possível constatar que os benefícios não atingem a parcela de trabalhadores mais pobres e em áreas rurais. Trabalhadores nessas condições tendem a ser excluídos desse tipo de benefício por questões burocráticas (dificuldades de pequenas empresas em participar de programas como o PAT), informalidade e menor organização sindical em relação à pauta de alimentação do trabalhador. Assim, esse tipo de benefício acaba concentrando seu atendimento nas regiões e estratos de maior renda. Um estudo realizado com trabalhadores no Estado da Bahia encontrou maior ocorrência do recebimento de benefícios alimentação e refeição na capital e nos estratos de renda mais elevados 15 .

Em relação ao estado nutricional da população beneficiária, verificou-se que a proporção de indivíduos com excesso de peso (53\%) foi um pouco superior à prevalência entre os adultos em geral $(50 \%$ entre os homens e $48 \%$ entre as mulheres) 20 . A preocupação em avaliar o estado nutricional dos usuários de benefícios alimentação e refeição é relevante para acompanhar o perfil antropométrico dos beneficiários e a qualidade da alimentação realizada fora do domicílio. Em 1996-1997, cerca de $32 \%$ dos beneficiários apresentavam excesso de peso e $24 \%$ tinham baixo peso 17 , um cenário bastante diferente do atual. Apesar da escassez de avaliações do impacto de programas de alimentação no estado nutricional de trabalhadores, um estudo conduzido no período de 1995-2000, na Bahia, verificou que trabalhadores de empresas credenciadas ao PAT e de empresas não credenciadas, mas que ofereciam refeições no local de trabalho, tíquete ou cesta básica, apresentavam as maiores taxas de incidência de ganho de peso e de sobrepeso em comparação com os trabalhadores não cobertos. Destaca-se que o ganho de peso e o sobrepeso foram maiores entre os trabalhadores de empresas não credenciadas ao PAT, mas cobertos por algum programa de alimentação 21 . No tocante à alimentação fora do domicílio, um estudo que comparou a qualidade nutricional de refeições consumidas em diferentes cenários verificou que as refeições feitas em restaurantes comerciais resultaram em maior consumo de gordura, proteína, doces e açúcar, quando comparadas àquelas realizadas no domicílio, e maior consumo de energia e menor ingestão de fibras, em relação às feitas no local de trabalho 22 .

Os benefícios alimentação e refeição, no âmbito do PAT, podem oferecer vantagens para as empresas, em determinadas situações. Esses benefícios permitem que pequenas empresas, que não têm condições para ter estruturas complexas como restaurantes dentro do ambiente de trabalho, participem do Programa. O benefício também permite que o trabalhador saia do ambiente de trabalho e realize suas refeições em conjunto com os colegas de trabalho, em ambientes apropriados 16, favorecendo a interação social no momento da refeição, algo valorizado para o alcance de uma alimentação saudável pelo Guia Alimentar para a População Brasileira ${ }^{23}$. Assim, essa modalidade de benefício tem o potencial de contribuir para a garantia da segurança alimentar e nutricional dos trabalhadores. No entanto, da forma como está distribuído, este potencial é limitado, pois, conforme mostrado no nosso estudo, está relacionado a trabalhadores residentes na Região Sudeste e em áreas urbanas que têm opções próximas ao local de trabalho para realizar suas refeições. A despeito do potencial dessa modalidade de benefício, seu valor deve ser suficiente para que o trabalhador possa realizar pelo menos uma refeição saudável 23 , considerando os dias trabalhados no mês. Ainda, ressalta-se a importância da realização de ações de educação alimentar e nutricional para os trabalhadores atendidos por essa modalidade.

As modalidades de benefício alimentação e refeição também recebem crítica pela forma de remuneração das empresas fornecedoras do benefício, uma vez que estas recebem, por parte das empresas que as contratam, e cobram uma taxa dos restaurantes credenciados. Assim, as empresas fornecedoras obtêm receita tanto dos restaurantes quanto das empresas contratantes, o que pode impactar no valor do benefício 24 .

A relevância deste estudo está relacionada à escassez de pesquisas sobre benefícios alimentação e refeição e à utilização de dados representativos da população brasileira. No entanto, este trabalho apresenta limitações. Acredita-se que é possível haver a subestimação de relato de recebimento desses benefícios, pois, por vezes, ele é recebido junto com o salário, sem um cartão específico, o que pode 
comprometer a referência por parte dos beneficiários. Ademais, a POF agrupa os benefícios refeição ealimentação, não permitindo avaliações separadas, ainda que estas modalidades possam impactar de forma diferente o consumo alimentar e a situação de segurança alimentar e nutriconal dos trabalhadores. Por fim, em função do período de referência para o recebimento do benefício (dados referentes ao valor recebido no mês anterior), para a compra domiciliar de alimentos e para a alimentação fora do domicílio (referentes aos últimos sete dias) serem diferentes, não foi possível avaliar o impacto do benefício na alimentação dos trabalhadores.
Apesar da tradição brasileira em políticas de alimentação e nutrição voltadas à população trabalhadora e ainda que decorram décadas de implantação do PAT, estudos avaliativos de âmbito nacional ainda são necessários 15,17,18. Utilizando os dados da POF foi possível estudar a abrangência dos benefícios alimentação e refeição no Brasil e o perfil dos beneficiários. Trata-se do primeiro estudo nacional que analisa essa informação, concluindo que uma importante parcela de trabalhadores está excluída do acesso a esses benefícios, e que mesmo entre aqueles incluídos são diversas as iniquidades regionais e sociais no acesso.

\section{Colaboradores}

D. S. Canella e D. H. Bandoni participaram do desenho, análise e interpretação dos dados e redação do texto. A. P. B. Martins contribuiu na interpretação dos dados e revisão crítica do texto.

\section{Agradecimentos}

D. S. Canella recebeu bolsa de pós-doutorado da Coordenação de Aperfeiçoamento de Pessoal de Nível Superior (Capes). 


\section{Referências}

1. Mondini L, Monteiro CA. Mudanças no padrão de alimentação na população urbana brasileira (1962-1988). Rev Saúde Pública 1994; 28:433-9.

2. Monteiro CA, Mondini L, Levy-Costa RB. Mudanças na composição e adequação nutricional da dieta familiar nas áreas metropolitanas do Brasil (1988-1996). Rev Saúde Pública 2000; 34:251-8.

3. Levy-Costa RB, Sichieri R, Pontes NS, Monteiro CA. Disponibilidade domiciliar de alimentos no Brasil: distribuição e evolução (1974-2003). Rev Saúde Pública 2005; 39:530-40.

4. Levy RB, Claro RM, Mondini L, Sichieri R, Monteiro CA. Distribuição regional e socioeconômica da disponibilidade domiciliar de alimentos no Brasil em 2008-2009. Rev Saúde Pública 2012; 46:6-15.

5. Goryakin Y, Surcke M. Economic development, urbanization, technological change and overweight: What do we learn from 244 Demographic and Health Surveys? Econ Hum Biol 2013; 14:109-27.

6. Bezerra IN, Souza AM, Pereira RA, Sichieri R. Contribution of foods consumed away from home to energy intake in Brazilian urban areas: the 20089 Nationwide Dietary Survey. Br J Nutr 2013; 109:1276-83.

7. Claro RM, Baraldi LG, Martins APB, Bandoni DH, Levy RB. Trends in spending on eating away from home in Brazil, 2002-2003 to 2008-2009. Cad Saúde Pública 2014; 30:1418-26.

8. Garcia RWD. Práticas e comportamento alimentar no meio urbano: um estudo no centro da Cidade de São Paulo. Cad Saúde Pública 1997; 13:455-67.

9. Ministério do Trabalho e Emprego. Programa de Alimentação do Trabalhador. Atlas do PAT: número de trabalhadores beneficiados por modalidade. http://consulta.mte.gov.br/atlas/deftohtm. exe?pat19.def (acessado em 06/Nov/2014).

10. Silva MHO. Programa de Alimentação do Trabalhador - PAT: estudo do desempenho de uma política social [Dissetação de Mestrado]. Rio de Janeiro: Escola Nacional de Saúde Pública, Fundação Oswaldo Cruz; 1998.

11. Associação Brasileira das Empresas de Refeições Coletivas. Mercado real. http://www.aberc.com. $\mathrm{br} /$ mercadoreal.asp?IDMenu=21 (acessado em 20/Fev/2015).

12. Instituto Brasileiro de Geografia e Estatística. Síntese de indicadores sociais: uma análise das condições de vida da população brasileira 2014. Rio de Janeiro: Instituto Brasileiro de Geografia e Estatística; 2014.
13. Instituto Brasileiro de Geografia e Estatística. Pesquisa de Orçamentos Familiares 2008-2009 - despesas, rendimentos e condições de vida. Rio de Janeiro: Instituto Brasileiro de Geografia e Estatística; 2010 .

14. World Health Organization. Physical status: the use and interpretation of anthropometry. Geneva: World Health Organization; 1995. (WHO Technical Report Series, 854).

15. Santos LMP, Araújo MPN, Martins MC, Veloso IS, Assunção MP, Santos SMC. Avaliação de políticas públicas de segurança alimentar e combate à fome no período 1995-2002. 2 - Programa de Alimentação do Trabalhador. Cad Saúde Pública 2007; 23:1931-45.

16. Wanjek C. Meal vouchers. In: Wanjek C, editor. Food at work: workplace solutions for malnutrition, obesity and chronic diseases. Geneva: International Labour Office; 2005. p. 153-98.

17. Burlandy L, Anjos LA. Acesso a vale-refeição e estado nutricional de adultos beneficiários do Programa de Alimentação do Trabalhador no Nordeste e Sudeste do Brasil 1997. Cad Saúde Pública 2001; 17:1457-64.

18. Ramos CA. Programas sociais: trajetória temporal do acesso e impacto distributivo. Brasília: Instituto de Pesquisa Econômica Aplicada; 2000. (Texto para Discussão, 77).

19. Instituto Análise. Pesquisa Refeição ASSERT Preço Médio 2012. http://assertbrasil.com.br/pesquisarefeicao-assert-preco-medio-2012/ (acessado em 02/Mar/2015).

20. Conde WL, Monteiro CA. Nutrition transition and double burden of undernutrition and excess of weight in Brazil. Am J Clin Nutr 2014; 100: 1617S-22S.

21. Veloso IS, Santana VS, Oliveira NF. Programas de alimentação para o trabalhador e ganho de peso e sobrepeso. Rev Saúde Pública 2007; 41:769-76.

22. Bandoni DH, Canella DS, Levy RB, Jaime PC. Eating out or in from home: analyzing the quality of meal according eating locations. Rev Nutr 2013; 26:625-32.

23. Ministério da Saúde. Guia alimentar para a população brasileira. 2a Ed. Brasília: Ministério da Saúde; 2014.

24. Robson R. Auctions in a two-sided network: the market for meal voucher services. Networks and Spatial Economics 2005; 5:339-50. 


\section{Abstract}

Food stamps and meal vouchers can determine workers' dietary choices. The study aimed to assess the coverage of these benefits in Brazil and their distribution according to the beneficiaries' socio-demographic and regional characteristics, using data from the Brazilian Household Budgets Survey, 2008-2009. Eligibility criteria were having an occupation and a private or government job, including domestic or temporary work in rural areas. Only 3.2\% of eligible individuals reported receiving such benefits. Highest coverage rates were verified with the Southeast region, urban areas, male gender, employment in the private sector, and monthly earnings $>$ five times the minimum wage. The mean monthly amount of such benefits was $R \$ 177.20$ (US\$100 at the 2009 exchange rate). After adjusting for other variables, the highest amounts were associated with male gender, higher salaries, the Northeast and Central regions, and employment in the public sector. This first analysis of the national coverage of food stamps and meal vouchers showed that a large share of Brazilian workers lack access or have unequal access to such benefits.

Food Assistance; Workers; Collective Feeding; Nutrition Programs and Policies; Social Inequity

\section{Resumen}

Considerando que la recepción de vales de alimentos y comida puede ser un factor determinante en la elección de la alimentación, el objetivo del estudio fue evaluar la cobertura de estos vales en Brasil y su asociación con las características sociodemográficas y regionales de los beneficiarios. Se utilizaron datos de la Encuestas de Presupuestos Familiares 2008-2009. Las personas que informaron tener una ocupación y declararon ser empleado privado, público, doméstico o temporal en zonas rurales fueron elegibles. Sólo el 3,2\% de los elegibles informaron haber recibido vales, con una mayor concentración en el sureste, área urbana, hombres, empleados en el sector privado y que reciben más de 5 salarios mínimos. El monto mensual promedio recibido de vales en Brasil fue de R\$177,20. Después de ajustar por otras variables, el valor fue mayor entre los hombres con salarios más altos en el noreste y medio-oeste y el sector público. Este es el primer análisis de la cobertura nacional en vales de alimentos y comida, destacando una parte importante de los trabajadores sin acceso a los vales y las inequidades en el acceso.

Asistencia Alimentaria; Trabajadores; Alimentación Colectiva; Programas y Políticas de Nutrición y Alimentación; Inequidad Social

Recebido em 11/Mar/2015

Versão final reapresentada em 09/Jun/2015

Aprovado em 01/Jul/2015 